\title{
A Morphological Classification Method of ECG ST-Segment Based on Curvature Scalle Space
}

\author{
Fusong $\mathrm{Hu}^{1,2}$, Chengtao $\mathrm{Li}^{2}$, Yongliang Zhang'2, Dan Jin², Zuchang $\mathrm{Ma}^{2}$, Yining Sun${ }^{2}$, \\ Jingzhi Wang1,2* \\ ${ }^{1}$ Department of Automation, University of Science and Technology of China, Hefei, China \\ ${ }^{2}$ Research Center for Information Technology of Sports and Health, Institute of Intelligent Machines, Chinese \\ Academy of Sciences, Hefei, China \\ Email: ${ }^{*}$ fortunepine@outlook.com
}

Received 24 July 2015; accepted 10 September 2015; published 17 September 2015

\begin{abstract}
Anomalous changes in the ST segment, including ST level deviation and ST shape change, are the major parameters in clinical electrocardiogram (ECG) diagnosis of myocardial ischemia. Automatic detection of ST segment morphology can provide a more accurate evidence for clinical diagnosis of myocardial ischemia. In this paper, we proposed a method for classifying the shape of the ST-segment based on the curvature scale space (CSS) technique. First, we established a reference ST set and preprocessed the ECG signal by using the CSS technique. Then, the corner points in the ST-segment were detected at a high scale of the CSS and tracked through multiple lower scales, in order to improve its localization. Finally, the current beat of ST morphology can be distinguished by the corner points. We applied the developed algorithm to the ECG recordings in European ST-T database and QT database to validate the accuracy of the algorithm. The experimental results showed that the average detection accuracy of our algorithm was $91.60 \%$. We could conclude that the proposed method is able to provide a new way for the automatic detection of myocardial ischemia.
\end{abstract}

\section{Keywords}

Myocardial Ischemia, Electrocardiogram, ST Shape Classification, Curvature

\section{Introduction}

Myocardial ischemia is a disorder of cardiac function. It occurs when the heart muscle, or myocardium, receives insufficient blood flow caused by a partial or complete blockage of the coronary arteries. Myocardial ischemia is a major cause of the occurrence of myocardial infarction and serious abnormal heart rhythms [1]. The major ECG symptom related with myocardial ischemia is ST segment change, including ST level deviation and ST

"Corresponding author.

How to cite this paper: Hu, F.S., Li, C.T., Zhang, Y.L., Jin, D., Ma, Z.C., Sun, Y.N. and Wang, J.Z. (2015) A Morphological Classification Method of ECG ST-Segment Based on Curvature Scale Space. Journal of Biosciences and Medicines, 3, 38-43. http://dx.doi.org/10.4236/jbm.2015.39006 
shape change. There has been increasing interest in automatic detection of ischemic ST-segment changes. A number of detection algorithms of ST segment change have been developed to detect ischemic episode, such as the artificial neural network [2] [3], the hidden Markov model [4], discrete wavelet transform (DWT) and support vector machine (SVM) [5]. However, most of these algorithms focused on the detection of the ST level deviation and only a few methods directly classify the ST shape. The curvature analysis has been widely used in locating the end of T-wave [6] and finding characteristic corner points of ECG [7]. The point of peak curvature can not only distinguish whether the current ST segment is straight line or curve, but also identify concave and convex. However, due to the influence of the noise and drift, the point of the peak curvature might not truly reflect the information of ST shape.

Mokhtarian et al. previously described a novel method for image corner detection based on the curvature scale-space (CSS) representation [8]. With the increase of scale, the noise is smoothed away and only the extreme corresponding to the real corners is remained. In this study, we introduced the CCS technique and developed an algorithm for ST shape classification. Firstly, we established a reference ST set that consists of five morphologies: horizontal, upsloping, downsloping, concave and convex [9]. Then we used the CSS technique to detect the corner points at a high scale, and tracked through multiple lower scales to improve localization. Finally, the point with the maxima of absolute curvature on ST-segment was used to classify ST shape. The ECG data in the European ST-T database and the QT database were used to validate the performance of the algorithm.

\section{Methods}

\subsection{Pre-Processing}

ECG signal is a weak low frequency and low amplitude voltages signal and can be easily affected by offsets and noise, such as power frequency interference, baseline wander, and Electromyographical interference. These noisy elements have to be removed before the signal is used for next analysis. In this study, we first used FIR (Finite Impulse Response) band-pass filter to remove the high frequency noise. The filtering frequency was set at 0.05 to $45 \mathrm{~Hz}$. Then, a Gaussian low-pass filter was applied to further smooth the ECG signal.

\subsection{Establishment of a Reference ST Set}

Before the experiment, a reference ST set was established. As shown in Figure 1, the morphology of ST was classified into five types: horizontal, upsloping, downsloping, concave, and convex. The aim of ST shape classification is to help physicians to distinguish an ischemic ST from a non-ischemic ST, and estimate how serious the ischemia is. For example, the emergence of the concave elevation often has no any significance or indicates pericarditis. However, convex and oblique straight elevation are showed to be more serious, and usually indicate acute myocardial infarction.

\subsection{The Curvature Scale-Space Technique}

The CSS technique was introduced by Mokhtarian et al. [8]. In that study, they proposed a novel method for image corner detection based on the CSS representation. This technique is suitable for standing out corner features at multiple scales. In the paper, we used this technique to extract the feature of ST segment. To compute it, the ECG signal is represented as follows:

$$
O(t)=(s(t), v(t))
$$

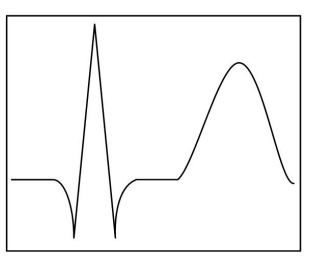

(a) Horizontal

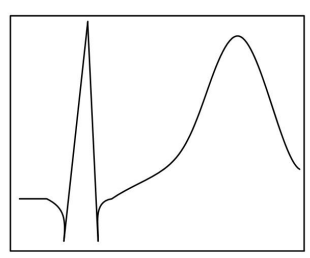

(b) Upsloping

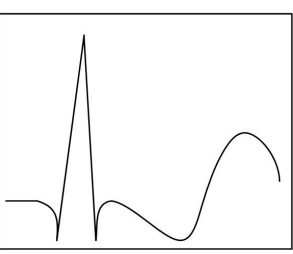

(c) Downsloping

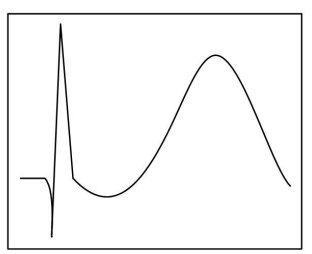

(d) Concove

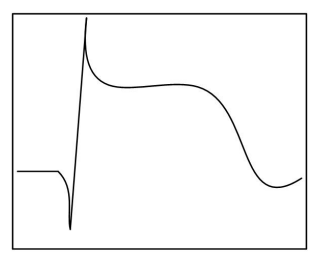

(e) Convex

Figure 1. A reference ST set. 
where $O(t)$ is the ECG original signal, $t$ is a time variable, $s(t)$ is a sample index at $t$ and $v(t)$ is a signal voltage. We converted $O(t)$ into the CSS by a Gaussian low pass filter and got the smoothed ECG signal $C(t, \sigma)$, defined by the following equation [7].

$$
C(t, \sigma)=(S(t, \sigma), V(t, \sigma))
$$

where $S(t, \sigma)=s(t) \otimes g(t, \sigma)$ and $V(t, \sigma)=v(t) \otimes g(t, \sigma) . g(t, \sigma)$ denotes a Gaussian function of width $\sigma . \otimes$ is the convolution operator. In this study, $\sigma$ is also taken as the scale parameter. The curvature $k(t, \sigma)$ is calculated based on the smoothed signal $C(t, \sigma)$ by Equation (3).

$$
k(t, \sigma)=\frac{S^{\prime \prime}(t, \sigma) V^{\prime}(t, \sigma)-S^{\prime}(t, \sigma) V^{\prime \prime}(t, \sigma)}{\left[S^{\prime}(t, \sigma)^{2}+V^{\prime}(t, \sigma)^{2}\right]^{3 / 2}}
$$

where $S^{\prime}(t, \sigma), S^{\prime \prime}(t, \sigma), V^{\prime}(t, \sigma)$, and $V^{\prime \prime}(t, \sigma)$ are expressed by Equation (4).

$$
\begin{aligned}
& S^{\prime}(t, \sigma)=s(t) \otimes \frac{\partial}{\partial t} g(t, \sigma), \\
& S^{\prime \prime}(t, \sigma)=s(t) \otimes \frac{\partial^{2}}{\partial t^{2}} g(t, \sigma), \\
& V^{\prime}(t, \sigma)=v(t) \otimes \frac{\partial}{\partial t} g(t, \sigma), \\
& V^{\prime \prime}(t, \sigma)=v(t) \otimes \frac{\partial^{2}}{\partial t^{2}} g(t, \sigma) .
\end{aligned}
$$

The curvature $k(t, \sigma)$ was used to extract the feature of ST segment and classify the morphology of ST segment. The corners correspond to the local maxima of the absolute curvature in the ST segment. There are many maxima in the ST segment due to noise at a low scale. With the increase of the scale, the noise is filtered and only the maxima that reflect the information about the ST shape remain.

\subsection{Feature Extraction of ST Segment}

Figure 2(a) shows an example of original ECG signal. The feature points including the peak of R wave and T wave, the start and the end of ST-segment were located by using the vertex selection method [7]. The start of ST-segment, also called J-point, is very important in locating the ST-segment and detecting the ST level. In order to eliminate the influence of noise on locating J-point, the concept of tracking in the multi-scale method based on the CSS was introduced to locate J-point. This process consists of the following 4 steps:

Step 1: The curvature of the smoothed ECG signal is calculated at the lowest scale $\sigma_{\text {lowest }}$.

Step 2: $R$ wave is detected by local extrema, which is located at the maximum voltage. $T$ wave is detected during the next 3/4 RR-interval. The extreme points in the range of [ $\left.R_{\text {peak }}+40 \mathrm{~ms}, R_{\text {peak }}+80 \mathrm{~ms}\right]$ are considered as the J-point candidates. The $R_{\text {peak }}$ expresses the peak of $R$ wave.

Step 3: Increase the scale to $\sigma_{\text {low }}$, and get a new curvature curve. The updated J-point candidates in the same range as Step 2 are obtained.

Step 4: To improve the location of the real J-point, tracing is introduced to the detection. Tracing is continued until the scale is very high $\left(\sigma=\sigma_{\text {high }}\right)$ and the new J-point candidates are obtained. The maximum point of the absolute curvature in the new J-point candidates is considered as the J-point.

As shown in Figure 2(b), the extreme point corresponding to the end of ST segment is not obvious. We located it by a supplemental vertex selection method [7].

\subsection{Morphological Classification of ST Segment}

ST shape classification is performed by calculating the vertex in ST segment that reflected the information about the bending degree of ST segment. However, due to the influence of the noise, the real vertex is difficult to detect. Therefore, we developed a rule by using the vertex in ST segment at multiple scales. The rule is as follows: 


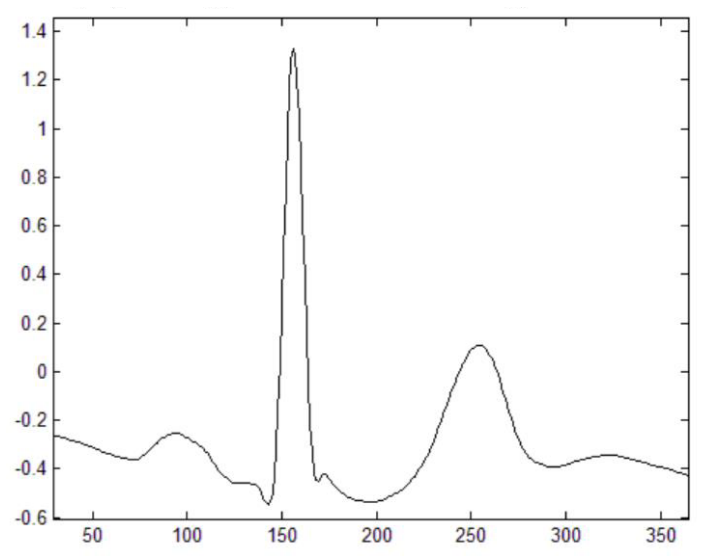

(a)

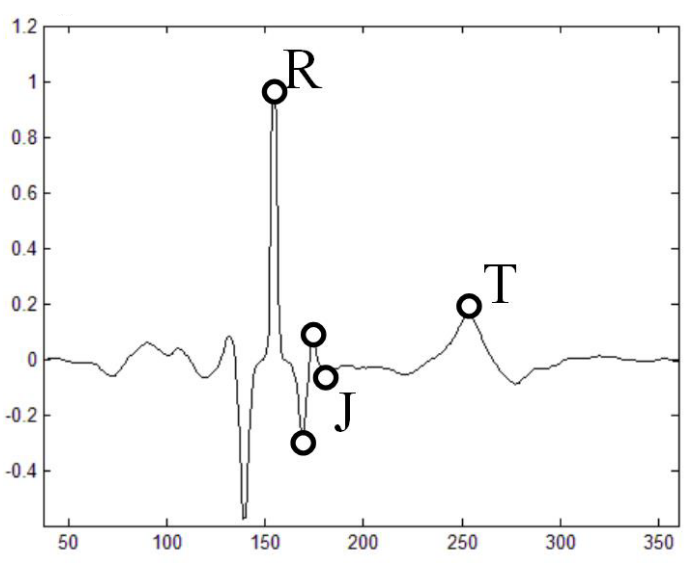

(b)

Figure 2. Original ECG signal (a) and the feature points of ST (b).

1) Calculate the mean and maximum curvature of ST segment at the scale $\sigma_{\text {low }}$. If the mean curvature of ST segment is within the threshold area $\left[-t_{\mathrm{avr}}, t_{\mathrm{avr}}\right]$ and the maximum curvature of ST segment is less than the threshold $t_{\max }$, the current ST is classified as linear shape. Otherwise the current ST is classified as curved shape.

2) If the voltage of the end of ST segment is greater than the start of ST segment for the linear shape of the ST segment, the current ST is classified as upsloping shape. Otherwise the current ST is classified as downsloping shape.

3) We find the point corresponding to the largest absolute curvature in the ST segment at the high scale $\sigma_{\text {high }}$ for the curved shape of the ST segment. This point is denoted by $K_{\text {cur }}$. If $K_{\text {cur }}>0$, the current ST is classified as convex shape. Otherwise that will be classified as concave shape.

\section{Experimental Results}

The ECG data in European ST-T database and QT database were used to evaluate the performance of the proposed algorithm. Thirteen ECG records were selected, 5 from European ST-T database and 8 from QT database. Analyses were performed using MATLAB (MATLAB 7.0, The Math Works Inc).

In order to determine the accuracy of the proposed algorithm, all the results were verified by a physician. Table 1 is the results of analysis for ECG records from QT database. The number of total heart beats is 983 . In general, the proposed algorithm showed an excellent classification performance. For sell03, se1114 and se 116272 ECG data, the correct rate of classification reached $100 \%$. The average correct rate is $96.54 \%$.

Table 2 shows the ST shape classification results for e0105, e0121, e0147, e0113 and e0207 ECG data from European ST-T database. The developed algorithm had the best classification result for e0121 record, with the correct rate of $98.89 \%$. The developed algorithm showed a poor performance for e0147 record. The correct classification rate is only $63.34 \%$. The reason may be that this record include the shape of upsloping, downsloping and horizontal etc. However, the average correct rate reached $86.66 \%$. The average classification accuracy of the proposed algorithm reached $91.60 \%$.

The proposed algorithm used two parameters (the scale $\sigma$ and threshold $t$ ) to extract the feature of ST segment and classify the ST shape. The experimental results showed that the algorithm had the best performance when $\sigma_{\text {lowest }}=6$ and the threshold $t_{\mathrm{avr}}=0.03$ and $t_{\max }=0.05$. If we start with $\sigma_{\text {lowest }}=4$, tracking can be accomplished at $\sigma_{\text {low }}=6$ and $\sigma_{\text {high }}=8-10$. Figure 3 shows the result of the ST shape classification by the proposed algorithm. In the figure, the start of the ST segment is marked with "ø" and the end of the ST segment is marked with " $\nabla$ ".

\section{Conclusions}

ST-segment change, including ST level deviation and ST shape change, is an important index for diagnosis of myocardial ischemia. The ST shape change can help physicians to find the real cause of ST change and provide a reference for diagnosis some diseases. Numerous automatic methods have been developed for detecting ST change in previous literature [2]-[4] [10]. However, only a few methods directly classify the ST shape. 
Table 1. The ST shape classification results of the QT database.

\begin{tabular}{ccccc}
\hline Record & No. of total beats & $\begin{array}{c}\text { No. of correctly } \\
\text { classified beats }\end{array}$ & $\begin{array}{c}\text { No. of wrong } \\
\text { classified beats }\end{array}$ & Correct rate (\%) \\
\hline sel103 & 243 & 243 & 0 & $100 \%$ \\
sel123 & 48 & 44 & 4 & $91.67 \%$ \\
sel100 & 80 & 78 & 2 & $97.5 \%$ \\
sel114 & 75 & 75 & 0 & $100 \%$ \\
sel14046 & 168 & 160 & 8 & $95.24 \%$ \\
sel16272 & 111 & 111 & 0 & $100 \%$ \\
sel14157 & 98 & 93 & 5 & $94.90 \%$ \\
sel223 & 160 & 145 & 15 & $90.63 \%$ \\
Total & 983 & 949 & 34 & $96.54 \%$ \\
\hline
\end{tabular}

Table 2. The ST shape classification results of the European ST-T database.

\begin{tabular}{ccccc}
\hline Record & No. of total beats & $\begin{array}{c}\text { No. of correctly } \\
\text { classified beats }\end{array}$ & $\begin{array}{c}\text { No. of wrong } \\
\text { classified beats }\end{array}$ & Correct rate (\%) \\
\hline e0105 & 90 & 79 & 11 & $87.78 \%$ \\
e0121 & 90 & 89 & 1 & $98.89 \%$ \\
e0147 & 90 & 57 & 33 & $63.34 \%$ \\
e0113 & 90 & 84 & 6 & $93.34 \%$ \\
e0207 & 90 & 72 & 8 & $80.00 \%$ \\
Total & 450 & 391 & 59 & $86.66 \%$ \\
\hline
\end{tabular}

(a) Horizontal shapes

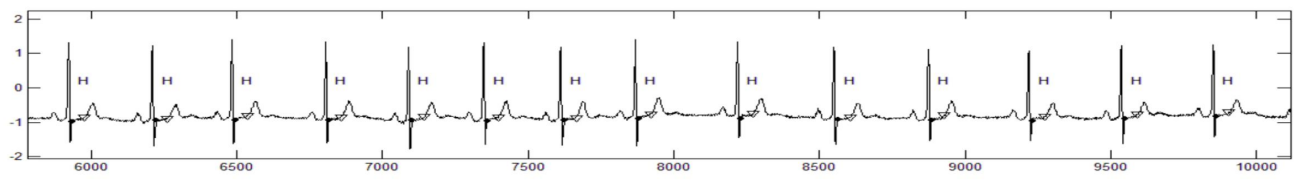

(b) Upsloping shapes

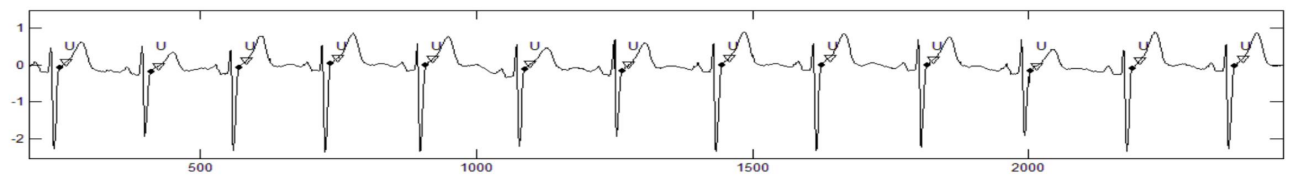

(c) Downsloping shapes

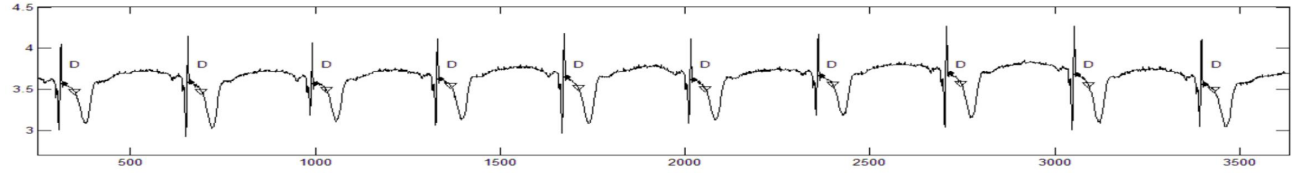

(d) Convex shapes

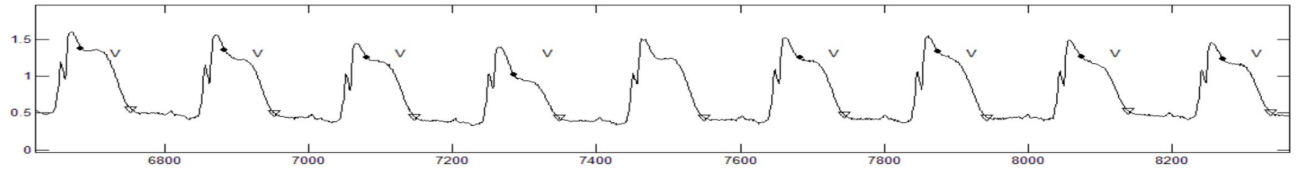

(e) Concave shapes

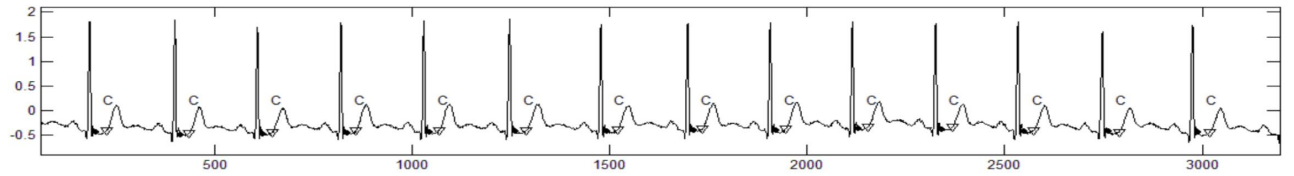

Figure 3. The result of the ST shape classification by the proposed algorithm: (a) Horizontal shape, (b) Upsloping shape, (c) Downsloping shape, (d) Concave shape, and (e) Convex shape. 
In this paper, we proposed a ST shape classification method based on the curvature scale-space technique. The point of absolute maximum curvature in ST segment was obtained by CSS technique and used to classify the ST shape. We applied the developed algorithm to European ST-T database and QT database. The results indicated that the proposed method has a high classification correct rate and is accurate in classifying ST shape. In addition, the proposed algorithm provides an effective method for the extraction of J-point, which can be used in extracting the start of the QRS complex as potential level. This method can be used to automatically classify the ST shape in long term ECG.

\section{Acknowledgements}

Fusong Hu and Chengtao Li contributed equally to this work. This work was supported by grant 61301059 from National Natural Science Foundation of China, 2013BAH14F01 from National Science and Technology Pillar Program and 1308085MF99 from the National Natural Science Foundation of Anhui Province.

\section{References}

[1] Garcia, J., Sornmo, L., Olmos, S. and Laguna, P. (2000) Automatic Detection of ST-T Complex Changes on the ECG Using Filtered RMS Difference Series: Application to Ambulatory Ischemia Monitoring. IEEE Transactions on Biomedical Engineering, 47, 1195-1201. http://dx.doi.org/10.1109/10.867943

[2] Gudipati, P. and Rajan, P.K. (2008) Ischemic Episode Detection in an ECG Waveform Using Discrete Cosine Transform and Artificial Neural Network. Proceedings of the 40th Southeastern Symposium on System Theory, New Orleans, 16-18 March 2008, 218-221. http://dx.doi.org/10.1109/ssst.2008.4480224

[3] Kwok, H.F., Giorgi, A. and Raffone, A. (2005) Improving Interpretability: Combined Use of LVQ and ARTMAP in Decision Support. Journal of Telecommunications and Information Technology, 129-132.

[4] Andreao, R.V., Dorizzi, B., Boudy, J. and Mota, J. (2004) ST-Segment Analysis Using Hidden Markov Model Beat Segmentation: Application to Ischemia Detection. Computers in Cardiology, 31, 381-384.

[5] Bustamante, C.A. and Duque, S.I. (2013) ECG Delineation and Ischemic ST-Segment Detection Based in Wavelet Transform and Support Vector Machines. Pan American Health Care Exchanges Conference (PAHCE), Medellin, 29 April-4 May 2013, 1-7. http://dx.doi.org/10.1109/pahce.2013.6568279

[6] Singh, Y.N. and Gupta, P. (2009) An Efficient and Robust Technique of T Wave Delineation in Electrocardiogram. Proceedings of the International Conference on Bio-Inspired Systems and Signal Processing, Porto, 14-17 January 2009, 146-154.

[7] Kim, T.H., Kim, S.Y., Kim, J.H., Yun, B.J. and Park, K.H. (2012) Curvature Based ECG Signal Compression for Effective Communication on WPAN. Journal of Communications and Network, 14, 21-26. http://dx.doi.org/10.1109/JCN.2012.6184547

[8] Mokhtarian, F. and Suomela, R. (1998) Robust Image Corner Detection through Curvature Scale Space. IEEE Transactions on Pattern Analysis and Machine Intelligence, 20, 1376-1381. http://dx.doi.org/10.1109/34.735812

[9] Jeong, G.Y., Yu, K.H., Yoon, M.J. and Inooka, E. (2010) ST Shape Classification in ECG by Constructing Reference ST Set. Medical Engineering \& Physics, 32, 1025-1031. http://dx.doi.org/10.1016/j.medengphy.2010.07.003

[10] Zimmerman, M.W. and Povinelli, R.J. (2004) On Improving the Classification of Myocardial Ischemia Using Holter ECG Data. Computers in Cardiology, 31, 377-380. http://dx.doi.org/10.1109/cic.2004.1442951 